\title{
ADJETIVOS DERIVADOS Y CUANTIFICACIÓN: LA HERENCIA DE RASGOS ASPECTUALES EN ESPAÑOL
}

\author{
LAURA KORNFELD \\ Universidad Nacional de General Sarmiento/ \\ Universidad de Buenos Aires/CONICET
}

\section{RESUMEN}

El presente trabajo se propone demostrar que en los adjetivos derivados de nombres y verbos se produce una herencia sistemática de una propiedad aspectual de la base: el rasgo [+/- delimitado]. Para ello hemos llevado a cabo un estudio de los fenómenos de cuantificación sintáctica que, como se ha observado repetidamente en la bibliografía relevante (cf. Bosque y Masullo 1997, Morimoto 1998), está directamente determinada por las características del aspecto léxico.

De este modo se sopesa la posibilidad de que la herencia de la delimitación sea análoga al bien estudiado fenómeno de la herencia de propiedades argumentales (cf., por ejemplo, Lieber 1983, 1992, Roeper 1987, Gracià 1995, Gracià y otros 1999, Rainer 1999, entre otros). Se trataría, pues, de un fenómeno que supone una compleja interacción entre diversos componentes de la gramática (morfología, sintaxis y léxico), más allá del modelo particular que se adopte.

Palabras clave: aspecto, delimitación, derivación morfológica, adjetivos, cuantificación.

\section{ABSTRACT}

This paper intends to demonstrate that in adjectives derived from nouns or verbs there is a systematic inheritance of an aspectual property of the base: the feature $[+/-$ delimited]. The analysis takes into consideration the phenomena of syntactic quantification which is directly determined by the characteristics of lexical aspect, as the relevant literature has observed (cf. Bosque y Masullo 1997, Morimoto 1998).

We consider the possibility that the inheritance of aspectual delimitation is a phenomenon analogous to the well-studied phenomenon of inheritance of argumental properties (cf., for instance, Lieber 1983, 1992, Roeper 1987, Gracià 1995, Gracià y otros 1999, Rainer 1999, among others). Independently of the particular model we adopt, the inheritance of aspectual delimitation would be, then, a phenomenon involving the interaction among different components of the grammar (morphology, syntax and lexicon).

Key Words: aspect, delimitation, morphological derivation, adjectives, quantification. 


\section{INTRODUCCIÓN ${ }^{1}$}

La cuantificación, y particularmente la gradualidad, constituye un campo poco explorado en la gramática del español, tal como ha notado Bosque 2001. El presente trabajo pretende precisamente contribuir a la investigación de las propiedades de la cuantificación, tomando en especial consideración el caso de los adjetivos, con el fin de determinar su relación con el aspecto léxico o Aktionsart. En concreto, nos proponemos demostrar que en los adjetivos derivados de nombres y verbos se produce sistemánticamente una herencia de una propiedad aspectual de la base: el rasgo [+/- delimitado]. Discutimos la posibilidad de que la herencia de la delimitación sea análoga al bien estudiado fenómeno de la herencia de propiedades argumentales (cf., por ejemplo, Lieber 1983, 1992, Roeper 1987, Gràcia 1995, Gràcia y otros 1999, Rainer 1999, entre otros).

Cabe señalar que los fenómenos empíricos que estudiamos aquí suponen una compleja interacción entre diversos componentes de la gramática (morfología, sintaxis y léxico), por lo que su análisis tiene interesantes repercusiones teóricas, con independencia del modelo particular que se adopte. Por otra parte, si bien los datos que se presentan provienen exclusivamente del español, es posible que las consecuencias del análisis alcancen también a los adjetivos derivados en otras lenguas.

En primer lugar (apartado II), haremos un breve repaso de la bibliografía que trata la relación entre aspecto léxico y cuantificación sintáctica, a fin de establecer los antecedentes relevantes para la investigación, principalmente Morimoto 1998, quien propone un rasgo transcategorial [+/- delimitado] para dar cuenta de una serie de oposiciones léxicas bien establecidas (verbos télicos y atélicos, adjetivos relacionales y calificativos, nombres contables y de masa), y Bosque y Masullo 1997, que han demostrado que existe una herencia del rasgo [+/- delimitado] en verbos denominales y deadjetivales. Después de revisar esos antecedentes, analizaremos en detalle (apartado III) cada uno de los casos de adjetivos derivados que suponen un cambio de categoría con respecto a la base: adjetivos deverbales (con significado activo y pasivo) y adjetivos denominales. En ambos casos intentaremos mostrar que las características aspectuales del adjetivo derivado (en particular, el rasgo [+/- delimitado], que define la posibilidad o imposibilidad de la cuantificación de grado), están determinadas fundamentalmente por las correspondientes propiedades de la base verbal o nominal. Es decir que los nombres y verbos con el rasgo [-delimitado] darán lugar a adjetivos con el mismo rasgo (que serán, por lo tanto, cuantificables), mien-

\footnotetext{
1 Quisiera agradecer los comentarios de los dos evaluadores anónimos que han leído la primera versión del artículo y me han permitido llegar a esta versión final y (espero) mejorada.
} 
tras que la presencia del rasgo [+delimitado] en las bases provocará que, al heredarlo, los adjetivos derivados no puedan recibir modificadores de grado.

\section{ESTADO DE LA CUESTIÓN}

\subsection{Aspecto léxico y cuantificación}

Una serie de oposiciones muy estudiadas en la bibliografía gramatical afecta a la interfaz léxico-sintaxis en la medida en que se corrobora que ciertas propiedades léxicas de los ítems influyen en su comportamiento oracional. Las diferencias de comportamiento entre los miembros de cada par se revelan claramente a partir de la cuantificación sintáctica.

Un primer contraste bien establecido, que se vincula tradicionalmente con el aspecto léxico o Aktionsart, se verifica entre los verbos atélicos, que se refieren a eventos básicamente homogéneos en su desarrollo (estados y actividades), y los verbos télicos, que tienen un punto culminante y son, por lo tanto, heterogéneos (logros y realizaciones) (cf. Vendler 1957, Dowty 1979, Morimoto 1998, entre otros). Ambos grupos de verbos presentan comportamientos muy disímiles en una serie de puntos; el que nos interesa aquí es, precisamente, la posibilidad de cuantificar el evento por medio de adverbios como mucho, demasiado, un poco, bastante. Esa cuantificación está limitada a los verbos atélicos ${ }^{2}$, como puede advertirse en los ejemplos de (1a) en contraste con los verbos télicos de (1b):

(1) a. corre mucho/la quiere demasiado/batió bastante la mezcla

b. *nace un poco/*construyó bastante una casa/*llegaremos demasiado

Otra oposición muy estudiada por la teoría léxica y sintáctica es la que se refiere a las distintas clases de adjetivos. Básicamente, los adjetivos calificativos o atributivos, que señalan una propiedad del nombre al que modifican, contrastan con otras dos clases: los adjetivos relacionales (de origen nominal, adscriben el nombre a una clase) y los adver-

\footnotetext{
${ }^{2}$ Cabe señalar que, pese a que la mayor parte de los verbos atélicos pueden ser cuantificados, esto no se aplica a algunos estados inacusativos:

(i) a. El chico está cansado (*mucho)

b. Los empleados sobran (*mucho)

c. La mercadería falta (*mucho)

Parece evidente que en los ejemplos de (i) se requiere una cuantificación sintáctica no verbal, sino de otra naturaleza (p. ej., muy cansado en (i.a), muchos empleados en (i.b) y mucha mercadería en (i.c.).
} 
biales (establecen circunstancias de modo, tiempo, aspecto o manera, de manera paralela a los adverbios en el ámbito oracional) (cf. Bosque 1990a, Bosque y Picallo 1996, Demonte 1999, entre otros). Nuevamente, la cuantificación brinda una pauta sintáctica de reconocimiento entre las distintas clases: los adjetivos calificativos pueden ser modificados por elementos de grado, como se observa en (2a). En cambio, no sucede lo mismo con los adjetivos relacionales (cf. 2b), ni con los adjetivos adverbiales que se refieren a tiempo y modo, como se ilustra en (2c) (los de manera y algunos adjetivos que señalan el aspecto, en cambio, parecen comportarse como simples calificativos: p. ej., un movimiento muy suave, un delito demasiado frecuente):

(2) a. un profesor muy lindo/nada inteligente/casi grandioso/medio tonto

b. un pozo (*bastante) petrolero/un sintagma (*demasiado) nominal/un animal (*re) acuático

c. el (*muy) futuro presidente/el (*bastante) aparente ladrón

Por último, es sabido que existen dos clases básicas de nombres comunes: los de masa, que señalan una sustancia sin límites definidos, y los contables, que señalan individuos (cf. Jackendoff 1991, entre otros). Quedaría fuera de los límites de este trabajo explicar todas las consecuencias de esta distinción, pero mencionaremos que cuantificadores como mucho, bastante, demasiado o poco deben aparecer en singular ante los nombres de masa y en plural ante los contables (cf. ejemplos 3-4a-b); que la modificación por medio de cuantificadores partitivos como algo/un poco de es propia de los nombres de masa (cf. ejemplos 3-4c), mientras que los numerales y otros determinantes son privativos de los contables (cf. ejemplos 3-4d); y que la aparición sin determinante en singular cuando funcionan como complementos de verbos y preposiciones es posible en los nombres de masa, e imposible en el caso de los contables (cf. ejemplos 3-4e):

(3) a. mucha/bastante/demasiada arena

b. *muchas arenas/*bastantes arenas/*demasiadas arenas

c. un poco/algo de arena.

d. *unas arenas $/ *$ tres arenas $/ *$ media arena $/ *$ cualquier arena $/ *$ toda arena

e. Compró café; un camión con madera

(4) a. *mucha revista/*bastante revista/*demasiada revista

b. muchas/bastantes/demasiadas revistas

c. *un poco/algo de revista

d. unas/tres revistas; media/cualquier/toda revista

e. Compró * (una) revista; una caja con * (una) revista (cf. Bosque 1999) 
Según la propuesta de Morimoto 1998, los contrastes ilustrados en (1-4) podrían ser reducidos a una sola oposición semántica básica: si el evento, propiedad o entidad tiene un límite (i.e., un punto culminante claro) o si se trata de un todo homogéneo sin límites, en el cual, por lo tanto, cualquier porción equivale a la totalidad. En esa visión, entonces, el par de rasgos transcategoriales [-delimitado] y [+delimitado] permite subsumir las diversas oposiciones reconocidas en la bibliografía entre verbos atélicos y télicos, entre adjetivos calificativos y relacionales (y también adverbiales) y entre nombres de masa y contables. A su vez, el comportamiento con respecto a la cuantificación sintáctica se desprendería directamente de la presencia del rasgo [+/-delimitado] en el ítem léxico: en el caso de adjetivos y verbos la posibilidad de cuantificación queda restringida a las formas con el rasgo [-delimitado]: adjetivos calificativos y verbos atélicos, según las clasificaciones tradicionales. En el caso de los nombres, en cambio, hay dos sistemas distintos de cuantificación, ilustrados en (3) y (4), que responden, respectivamente, a nombres con el rasgo [-delimitado] (de masa) y nombres con el rasgo [+ delimitado] (contables).

\subsection{Herencia del aspecto}

Como se ha anticipado en la introducción, en este artículo se pretende analizar el caso de los adjetivos derivados morfológicamente, intentando determinar si es plausible que exista la herencia de un rasgo aspectual como [+/-delimitado] de un modo análogo a lo que se verifica en relación con la herencia de propiedades argumentales. Cabe recordar que la herencia argumental se refiere a la transmisión de propiedades de la base al producto en la derivación morfológica. En la bibliografía pertinente no hay acuerdo acerca del modo más adecuado de reflejar cómo se produce la herencia de los requerimientos argumentales del verbo, en la medida en que tampoco está claro si la estructura argumental en sí es una propiedad semántica o sintáctica de los ítems. Algunos autores proponen que lo que se hereda es una propiedad sintáctica (cf. Hale y Keyser 1993, Gràcia 1995, por ejemplo) y otros, que se trata un rasgo semántico (cf. De Miguel 1986); a los fines de nuestro interés en este artículo, esa discusión no tiene mayor relevancia.

Algunos ejemplos concretos del fenómeno de herencia argumental se presentan en los pares de (5) y (6), que ilustran casos en los que un nombre o un adjetivo hereda los requerimientos argumentales de su base verbal:

(5) a. EE.UU. vendió armas a Irán durante muchos años

b. La venta de armas a Irán (por parte de EE.UU.) resultó un fracaso 
(6) a. Ese concepto se aplica a esta situación

b. Es un concepto aplicable a esta situación

Las palabras derivadas de los ejemplos (b) exhiben propiedades relativamente «insólitas» para su categoría en cuanto a la selección de los complementos: los diversos complementos preposicionales para venta en (5b) (i.e., el tema precedido por $d e$, el benefactivo por $a$ y el agente) o el complemento preposicional para aplicable en (6b) (que, al igual que en el caso del verbo debe estar encabezado por la preposición «regida» $a$ ).

Como antecedente de la idea de que la delimitación aspectual pueda heredarse en la derivación morfológica, debe señalarse el estudio de Bosque y Masullo 1997 sobre la cuantificación verbal. Basándose en una particular aplicación del modelo de Hale y Keyser 1993, Bosque y Masullo analizan el comportamiento de verbos denominales y deadjetivales en relación con la cuantificación, partiendo del supuesto de que no existe la cuantificación verbal per se, sino que la presencia de cuantificadores como un poco, bastante o mucho se debe a la modificación de un elemento presente en la estructura léxico-relacional (ELR) del verbo. Según Bosque y Masullo, en los verbos de (7-8.a), que admiten la cuantificación ${ }^{3}$, el cuantificador modifica en realidad al nombre involucrado en la ELR, que es sistemáticamente un nombre de masa: enmantecar/aceitar, azucarar/salar un poco se parafrasearían como "poner un poco de manteca/aceite /azúcar/sal» y llorar/reir/brillar/nevar mucho como «hacer mucho llanto/risa/ brillo/nieve» ${ }^{4}$. Al ser contables, los nombres que sirven de base a los verbos de (7-8b) no admiten la cuantificación, ya que incluso las eventuales paráfrasis carecerían de sentido: «*/?? poner un poco de muralla/alfombra/silla», «*/?? pasar bastante verano/invierno/vacaciones», etcétera.

(7) a. enmantecar/aceitar/azucarar/salar/adornar/alquitranar un poco

b. amurallar/ alfombrar/ensillar/cercar/forrar/adoquinar (*un poco)

(8) a. llorar/reír/brillar/nevar/trabajar/bailar bastante

b. veranear/invernar/vacacionar (*bastante)

\footnotetext{
3 «Cuantificación» se usa aquí, exclusivamente, en el sentido de cuantificación inherente, que se diferencia de la cuantificación aspectual (viene mucho a casa) y temporal (esperó mucho).

${ }^{4}$ Puntualmente, según el análisis de Bosque y Masullo, esos verbos tendrían en su ELR un SQ (Sintagma de Cuantificación) vinculado con un elemento presente en la estructura argumental del verbo. No me detendré aquí a criticar en detalle esta solución que me parece teóricamente defectuosa y además incompatible con el marco de Hale y Keyser, quienes suponen que en las ELR o estructuras argumentales sólo pueden participar elementos léxicos, y nunca categorías funcionales.
} 
Más allá del análisis puntual de Bosque y Masullo, nos interesa destacar que en su estudio muestran que un número relevante de verbos denominales y deadjetivales aceptan o no la cuantificación sintáctica de acuerdo con el rasgo [+/- delimitado] de la base. Así pues, es la presencia o ausencia del rasgo en la base léxica la que explicaría la diferencia de comportamiento entre verbos de estructuras semejantes: verbos de locatum en (7) y verbos inergativos en $(8)^{5}$. Admiten ser cuantificados, entonces, los verbos que se derivan de nombres de masa con el rasgo [-delimitado], como los de (7-8a), pero no los que provienen de nombres contables que tienen el rasgo [+delimitado], como los de (7-8b).

Una distribución similar observan Bosque y Masullo con respecto a los verbos deadjetivales que admiten la cuantificación (en general, verbos ergativos que permiten la alternancia de variantes transitiva-inacusativa): se derivan sistemáticamente de adjetivos con el rasgo [-delimitado], esto es, adjetivos calificativos que también admiten la cuantificación de grado, como muestran los ejemplos de (9):
(9) a. ensanchar/acortar/engordar/adelgazar/ennegrecer/aclarar mucho
b. muy ancho/corto/gordo/delgado/negro/claro ${ }^{6}$

\section{ANÁlisis DE lOS ADJETIVOS DERIVADOS}

\subsection{Propósito}

En esta sección nos ocupamos de estudiar las propiedades de los dos grupos de adjetivos derivados que agotan las posibilidades de derivación con cambio categorial: los adjetivos deverbales y los denominales. Puntualmente, analizamos su comportamiento frente a la cuantificación, es decir, si aceptan o no modificadores de grado como muy, bastante, poco ${ }^{7}$, etc. o, en términos más teóricos, si pueden ser tomados como complementos de un Sintagma de Grado (de acuerdo con la formulación más

\footnotetext{
${ }^{5}$ Los verbos de locación como enjaular, encarcelar, embarcar, acorralar, en cambio, se derivan sistemáticamente de nombres con el rasgo [+delimitado], según observan Bosque y Masullo 1997, p. 50, por lo que no admiten nunca la cuantificación sintáctica.

${ }^{6}$ Si bien no son muy frecuentes, verbos como los de (i) se forman sobre adjetivos relacionales, una posibilidad no contemplada en Bosque y Masullo. Tal como es esperable, mantienen el aspecto de la base nominal (tienen también el rasgo [+delimitado]), por lo cual no pueden ser cuantificados:

(i) a. nominalizar/adjetivar/verbalizar/criminalizar (*un poco) b. (*muy) nominal/adjetivo/verbal/criminal

7 Descartamos, en cambio, otros elementos de Grado por considerar que tienen restricciones ulteriores y no se aplican a todos los adjetivos graduables: por ejemplo, medio, un tanto, un poco: *medio inteligente/astuto/veloz (cf. Kornfeld 2007).
} 
o menos estándar adoptada por la gramática generativa desde los años 90). Ponemos en relación esa propiedad con dos características de las palabras de las que se derivan: el rasgo [+/-delimitado] y el comportamiento sintáctico frente a la cuantificación.

Es conveniente resaltar que en esta sección de análisis dejamos de lado los casos de derivación homogénea (esto es, sin cambio de categoría), ya que en su amplia mayoría se trata de procesos que involucran la afijación apreciativa. Esos procesos, por definición, implican siempre la conservación del rasgo [-delimitado] de la base en el resultado. En efecto, la afijación apreciativa solo puede aplicarse a adjetivos graduables: mientras que son posibles formas creadas por sufijación de adjetivos calificativos (como triste/tristón, chico/chiquito, grande/grandote/grandísimo, etc.), no lo son las basadas en adjetivos relacionales (como nominal/*nominalísimo y petrolero/* petrolerote) o adverbiales (como aparente/*aparentito y futuro/*futurón). Lo mismo se aplica a los adjetivos derivados por prefijación (que por definición no implican cambio categorial), por lo que solo nos referimos a ellos en forma marginal, cuando se combinan con ciertos sufijos deverbales como -ble.

\subsection{Adjetivos deverbales}

En este apartado evaluamos la posibilidad de que haya herencia de la delimitación en el caso de los adjetivos deverbales, analizando las propiedades de bases y derivados. Estos adjetivos a menudo han sido dejados de lado en la dicotomía entre adjetivos calificativos y relacionales, ya que -como discutimos en detalle- no se ubican «en bloque» en ninguno de los dos grupos.

Diferenciamos aquí dos tipos diferentes de adjetivos deverbales: adjetivos con significado activo (o, en otros términos, que modifican al agente o argumento externo del verbo base) y adjetivos con significado pasivo -que modifican al tema o argumento interno del verbo base-, de acuerdo con la distinción sostenida por la bibliografía que se ocupa del fenómeno de herencia argumental (cf., sobre todo, Gràcia 1995 y Gràcia y otros 1999). En cada caso, comparamos las posibilidades de cuantificación de los tipos de adjetivos con las de sus correspondientes bases verbales.

$\mathrm{Al}$ igual que Bosque y Masullo, para los verbos solo tomamos en cuenta la cuantificación inherente, dejando de lado los casos en los que los cuantificadores tienen un valor exclusivamente aspectual (=«seguido», como en viene mucho) o temporal (= «mucho tiempo», como en esperó mucho). También descartamos los posibles usos pronominales o argumentales del cuantificador, como en aprendió/compró/vendió/ganó mucho (en los que el cuantificador está en lugar del argumento requerido 
por el verbo), incluyendo los casos de verbos bivalentes como leyó/escribió/comió/bebió mucho, donde el cuantificador puede modificar ambiguamente a la lectura transitiva o intransitiva del verbo.

\subsubsection{Adjetivos deverbales con significado activo}

Entre los adjetivos deverbales con significado activo que modifican al nombre que funciona como agente o argumento externo del verbo base, se encuentran los conformados por los sufijos -dor y -nte y por otros morfemas menos frecuentes, como -ón, -ivo o -izo.

El sufijo -dor se combina sólo con verbos inergativos y transitivos y es incompatible con verbos inacusativos ${ }^{8}$. Se ha señalado que los adjetivos derivados con ese sufijo tienen dos paráfrasis posibles, siempre de interpretación agentiva, en relación con las correspondientes bases verbales. En un caso, los adjetivos se parafrasean como «que V mucho» (trabajador/caminador/seductor/salidor/participador/conversador); en el otro, reciben la interpretación «que V» (descubridor [de América]/ganador [de la carrera]/cazador [de ratones] / constructor [de casas]). $\mathrm{Al}$ analizar los dos grupos, se advierte que la primera interpretación es la predominante entre los adjetivos que provienen de verbos atélicos y la segunda, en las formas creadas a partir de verbos télicos (cf. Gràcia y otros 1999, Rainer 1999).

Ambos grupos se comportan diferenciadamente respecto de la cuantificación: mientras que los adjetivos que reciben la primera interpretación pueden recibir modificadores de grado (que, de hecho, están incluidos en la propia paráfrasis «que V mucho»), los que responden a la segunda no pueden:

(10) a. un hombre muy trabajador/caminador/seductor/salidor/participador/conversador

b. la expedición (*muy) descubridora de América/el potrillo (*muy) ganador de la carrera/un gato (*muy) cazador de ratones

El contraste entre (10a) y (10b) puede explicarse siguiendo los argumentos que ya hemos esbozado. Si se analiza la posibilidad o imposibilidad de que los verbos de base reciban cuantificadores verbales con interpretación inherente, referida al evento, se comprueba que el carácter [+/-delimitado] de los verbos de base es heredado por el adjetivo. En (11) se presentan los verbos de base de los adjetivos derivados de (10), que muestran una reacción perfectamente paralela con respecto a la cuantificación sintáctica:

\footnotetext{
${ }^{8}$ No son excepciones a esta generalización formas como entrador, salidor, llegador, faltador, ya que refieren sistemáticamente al significado agentivo de cada verbo.
} 
(11) a. El hombre trabaja/camina/seduce/sale/participa/conversa mucho

b. La expedición descubrió (*mucho) América./El potrillo ganó (*mucho) la carrera./El gato cazó (*mucho) ratones

En otros términos, los adjetivos que admiten la cuantificación de grado se derivan sistemáticamente de verbos atélicos que también admiten ser cuantificados, como muestran los ejemplos (10-11a). Consecuentemente, tanto adjetivos como verbos base comparten el rasgo [-delimitado]. Lo contrario puede decirse de los casos de (10-11b): los verbos télicos, con el rasgo [+delimitado], dan lugar a adjetivos no graduables. La correlación es, pues, paralela a la que señalan Bosque y Masullo 1997 para los verbos denominales, que ya hemos presentado en la sección 2.2.

Por su parte, los adjetivos deverbales en -nte comparten su paráfrasis con los adjetivos en -dor: «que V». Aunque alterna con -dor en la combinación con verbos agentivos, transitivos o inergativos (cf., por ejemplo, contaminante, refrescante, cicatrizante), el sufijo-nte se distingue porque puede adjuntarse también a verbos inacusativos y estativos, como en naciente, muriente, saliente, faltante, sobrante, etc. (cf. Laca 1993). Tal como era de esperar, de acuerdo con lo que hemos visto aquí, en cualquiera de los dos tipos de combinación, admiten modificación de grado los adjetivos en -nte que se derivan de verbos que pueden ser cuantificados (i.e., verbos atélicos con el rasgo [-delimitado $]^{9}$ ), mientras que lo contrario ocurre con los adjetivos formados sobre bases télicas. Los ejemplos de (12) muestran ese funcionamiento sintáctico diferenciado, mientras que los de (13) ilustran cómo se comportan con respecto a la cuantificación los verbos de base correspondientes:

(12) a. Era muy vigilante/cambiante/desafiante/obediente/contaminante/ refrescante

b. Es (*muy) naciente/renunciante/rompiente/iniciante/cicatrizante

(13) a. Vigila/cambia/desafía/obedece/contamina/refresca mucho

b. Nace/renuncia/se rompe/inicia/cicatriza (*mucho)

Hay, pues, una consistencia entre los adjetivos en -nte de (12a) y los verbos base de (13a), por un lado, y los de (12b) y (13b) por el otro, en

\footnotetext{
adjetivos derivados:
(i) a. Los empleados sobran (*mucho)
b. *La mercadería falta mucho
(ii) a. los empleados (*muy) sobrantes

b. la mercadería (*muy) faltante
}

${ }^{9}$ Como hemos advertido en la nota 2 , ciertos estados inacusativos como faltar o sobrar no pueden cuantificarse, si bien son [-delimitados]. Consecuentemente, lo mismo sucede con los 
forma paralela a lo que hemos visto para los adjetivos en -dor. Nuevamente, se deduce de estos ejemplos que el adjetivo siempre hereda el rasgo [+/-delimitado] de la base y que ese rasgo determina si admite, o no, la cuantificación de grado.

Por su parte, otros sufijos con un significado activo análogo a -dor y -nte solo pueden adjuntarse a verbos atélicos, es decir, con el rasgo [-delimitado] y solo dan lugar, por lo tanto, a adjetivos cuantificables. Entre ellos cabe mencionar los casos de los sufijos -ón, -ivo o-izo. Mientras que los adjetivos de (14a) reciben la paráfrasis «que V mucho», con valor eminentemente apreciativo, los de (14b) se interpretan como «que $\mathrm{V}$ » y los de (14c) como «que (se) V fácilmente», intermedio en relación con los adjetivos de significado pasivo+modal, como -ble (cf. próximo apartado):

(14) a. muy tragón/mirón/burlón/reclamón ( $\rightarrow$ 'que traga/mira/burla/reclama mucho')

b. muy expresivo/ofensivo/pensativo ( $\rightarrow$ 'que expresa/ofende/ piensa mucho')

c resbaladizo/enamoradizo/quebradizo ( $\rightarrow$ 'que se resbala/se enamora/se quiebra mucho')

\subsubsection{Adjetivos deverbales con significado pasivo}

En este apartado analizamos los adjetivos deverbales con significado pasivo, es decir, aquellos que, desde el punto de vista argumental, modifican al nombre que funciona como tema o argumento interno del verbo base, como los participios convertidos en adjetivos o los adjetivos formados con el sufijo -ble.

La creación de adjetivos a partir de participios verbales es uno de los fenómenos más productivos de conversión categorial, no sólo en espanol sino en las lenguas indoeuropeas en general ${ }^{10}$. Una restricción relevante que se aplica a diversas lenguas es que los verbos inergativos no pueden dar lugar a adjetivos participiales (entendidos como las formas participiales que tienen la misma distribución y las mismas propiedades que los adjetivos). La imposibilidad de formar adjetivos participiales se debe a que los verbos inergativos carecen de argumento interno; de todos modos, ese primer requisito no basta para explicar la conversión, puesto que los adjetivos participiales tampoco pueden formarse a partir de cualquier verbo inacusativo o transitivo (cf. Bosque 1999b).

${ }^{10}$ Si bien podría objetarse la pertinencia de estudiar en este artículo el caso de los adjetivos participiales -en la medida en que se trata de un caso de conversión, y no de derivación morfológica-, cabe recordar que esos adjetivos han sido tratados como deverbales en diversos estudios sobre herencia argumental, incluyendo los artículos clásicos de Bresnan 1978 o Levin y Rappaport 1986. 
Una de las propiedades que indica si una forma participial tiene el funcionamiento propio de un adjetivo es, precisamente, la posibilidad de tomar un modificador de grado. Los ejemplos de (15) muestran un claro contraste en la posibilidad de que distintas formas participiales, que encabezan modificadores nominales, acepten un cuantificador $^{11}$ :

(15) a. un hombre muy aplaudido/llorado/apabullado/cansado/lastimado

b. un hombre (*muy) nacido/muerto/desembarcado/llegado

De acuerdo con lo que hemos discutido hasta aquí, resulta por lo menos dudoso considerar que la propiedad de aceptar cuantificadores de grado sea per se definitoria de la clase adjetivo, dado que hemos comprobado que numerosos adjetivos conformados por los sufijos -dor o -nte, por ejemplo, no los admiten. Sin embargo, en el caso de las formas participiales que no aceptan la aparición del Sintagma de Grado (cf. 15b), se verifica fácilmente que conservan su significado eventivo original y que a menudo deben ir acompañados de complementos y adjuntos típicamente verbales, como se ilustra en (16):

(16) a. un hombre nacido/muerto/desembarcado llegado en 1979

b. un edificio construido por los obreros/atribuido a Da Vinci

En cuanto a las formas participiales que admiten la cuantificación de grado (15a), conviene aclarar que suelen ser ambiguas desde el punto de vista categorial (cf. 17a), cuando carecen de un contexto claro, sea adjetivo (cf. 17b) o verbal (cf. 17c):

(17) a. una chica pintada/una empresa arruinada

b. una chica muy pintada/una arruinadísima empresa

c. una chica pintada en un santiamén por su mamá/una empresa arruinada en los años '90 por las políticas neoliberales

11 Entre las formas participiales que admiten la modificación de grado (cf. 15a), algunas aceptan el cuantificador ya en la perífrasis pasiva (cf. ia), mientras que otras no (cf. i.b):

(i) a. fue muy/poco aplaudido/celebrado/llorado/conocido/querido/apreciado

b. *fue muy/poco pintado/cansado/quebrado/arruinado/arreglado

Bosque 1999b observa que los cuantificadores muy, tan, bastante o demasiado en ejemplos como (i.a) están modificando formas participiales de evidente naturaleza verbal. Por lo tanto, propone, como prueba más contundente de la conversión de la forma participial, la posibilidad de adjunción del afijo diminutivo -it $(o / a)$, exclusiva de la categoría adjetivo. Sin embargo, es preciso recordar que el diminutivo se aplica solo a un subconjunto de los adjetivos graduables: p. ej. *inteligentito, *velocito, *astutito (cf. Kornfeld 2007), por lo que la imposibilidad de adjunción del afijo diminutivo puede deberse a diferentes motivos y no sólo a la naturaleza verbal de la forma (cf., de hecho, *conocidito o *aplaudidito). 
Nótese que la presencia del elemento de grado es incompatible con la aparición de modificadores típicamente verbales, es decir, los contextos de $(17 b)$ y (17c) son mutuamente excluyentes, como se comprueba en (18):

(18) *una chica muy pintada en un santiamén por su mamá/*una arruinadísima empresa en los años '90 por las políticas neoliberales

Ahora bien, si volvemos al contraste inicial de (15), veremos que la diferencia de comportamiento respecto de la cuantificación de grado se puede atribuir, una vez más, a la presencia del rasgo [+/-delimitado] en la base verbal. Mientras que los verbos de base de los ejemplos de (15a) admiten la cuantificación inherente, puesto que se trata de verbos no delimitados, como muestra (19a), los de (15b) no la admiten, ya que son verbos télicos, es decir, delimitados, como muestra (19b):

(19) a. Aplaude/llora/apabulla/se cansa/se lastima mucho

b. Nace/muere/construye/desembarca/llega (*mucho)

Cabe resaltar que la posibilidad de cuantificación del verbo implica necesariamente que el evento es concebido como [-delimitado], ya que, al cuantificar el verbo, suponemos que no hay un punto culminante que deba atravesarse para dar el evento por cumplido. Esa afirmación resulta válida incluso para ciertos verbos que pueden clasificarse como realizaciones (i.e., eventos télicos), como los casos de adjetivos participiales y sus bases verbales que se presentan en (20-21):

(20) una chica muy pintada/perfumada/arreglada/quebrada/destruida

(21) a. Se pintó/perfumó/arregló mucho.

b. La noticia la quebró/destruyó bastante

Nótese que los verbos de base de los adjetivos participiales de (20) exhiben, en principio, los comportamientos típicos de las realizaciones; así, es posible combinarlos con modificadores aspectuales télicos: se pintó/se quebró en un minuto/una hora (cf. Vendler 1957). Sin embargo, los ejemplos con cuantificadores de (21) demuestran que también es posible focalizar los eventos en su desarrollo como si fueran atélicos, en cuyo caso resultan incompatibles con esos modificadores: se pintó mucho/se quebró bastante (*en un minuto/una hora).

En suma, las posibilidades de conversión del participio en adjetivo dependerán de la presencia del rasgo [-delimitado] en la base, ya que, como ilustran los contrastes anteriores, sólo en el caso de las formas participiales creadas sobre verbos no delimitados podrán aparecer los modificadores de grado que se consideran distintivos de la categoría adje- 
tivo (cf. Bosque 1990b, 1999b, Kornfeld y Resnik 2002) ${ }^{12}$. Por lo tanto, la predicción es que todos los adjetivos formados por conversión de la forma participial aceptarán, necesariamente, la cuantificación de grado.

El comportamiento de los adjetivos en -ble, en cambio, resulta menos sencillo de anticipar. Es posible, empero, formular dos generalizaciones relevantes: los adjetivos provenientes de verbos atélicos, con el rasgo [-delimitado], siempre son graduables (compárense los adjetivos graduables de 22a con los verbos base de 23a), mientras que los adjetivos que no permiten la cuantificación se derivan todos de bases télicas, con el rasgo [+delimitado] (cf. 22b y 23b).

(22) a. muy comprensible/acumulable/inflamable/querible/vulnerable/ deseable

b. (*muy) armable/caracterizable/aplicable/evitable/sustituible

(23) a. comprender/acumular/querer/vulnerar/inflamar/desear mucho b. armar/caracterizar/aplicarse/evitar/sustituir (*mucho)

Ahora bien, la posibilidad de recibir cuantificadores de grado en los adjetivos en -ble está más extendida de lo que sería esperable tomando en cuenta el rasgo de la delimitación de la base verbal, como puede advertirse en los ejemplos de (24):

(24) a. muy concebible/reparable/demostrable/navegable

b. concebir/reparar/demostrar/navegar (*mucho)

Una explicación plausible para el hecho de que los adjetivos graduables de (24a) se deriven de verbos que no admiten la cuantificación inherente como los de (24b) se vincula con la reinterpretación semántica que puede realizarse a partir del elemento modal implícito en la paráfrasis de los adjetivos en -ble. En efecto, se ha señalado que el significado que aporta el sufijo -ble a la base verbal debería parafrasearse, en principio, como «que puede ser Vdo», pero que a menudo el adjetivo se reinterpreta como «fácil de V»(Gràcia 1995). Esta lexicalización sistemática explicaría que existan adjetivos como los de (24a) que se derivan de verbos delimitados: la cuantificación del adjetivo se leerá en estos ejemplos como «muy fácil de V» y no como «fácil de V mucho».

De este modo, un número de formas en -ble que, por derivarse de verbos delimitados, no deberían poder ser cuantificadas por elementos de grado, admiten esos modificadores en función de un patrón sistemático de lexicalización, que se verifica en la paráfrasis.

\footnotetext{
${ }^{12}$ Evidentemente, los verbos inergativos (en su mayoría con el rasgo [-delimitado]) no suelen dar lugar a adjetivos participiales por motivos independientes (i.e., carecen en general de argumento interno, según la explicación de Levin y Rappaport 1986, Bosque 1990b, 1999b, Kornfeld y Resnik 2002, entre otros).
} 
Las generalizaciones establecidas previamente se aplican también a los adjetivos en -ble conformados por el prefijo de significado negativo in-, que pueden tener una frecuencia de aparición mucho mayor que las correspondientes bases (indestructible, ineludible) o incluso carecen de contrapartes positivas (indudable). La paráfrasis «literal» para estas formas es «que no puede ser Vdo», pero a menudo hay una lexicalización análoga a la de los correspondientes adjetivos positivos, en la que el significado negativo del prefijo modifica a «fácil» dando lugar a la paráfrasis «difícil de V». Cuando aparece el cuantificador de grado modifica entonces a ese elemento modal implícito («muy difícil de V»). La lexicalización es independiente de las propiedades aspectuales del verbo base, como se puede advertir en la comparación de los adjetivos de (25) con los verbos correspondientes de (26), que muestra que, mientras que algunos adjetivos cuantificables (cf. 25a) se derivan de verbos con el rasgo [-delimitado] (cf. 26a), otros no lo hacen (cf. 25b-26b), en forma análoga a los pares de (24). Por su parte, los adjetivos no cuantificables de (25c) sí se derivan, esperablemente, de los verbos télicos de (26c), en forma paralela a $(22-23 b)^{13}$ :

(25) a. muy/demasiado increíble/insoportable/incomprensible/invulnerable

b. muy/demasiado inconcebible/irreparable/indemostrable/indescriptible

c. ?? muy/demasiado insustituible/inevitable/irrevocable/insolucionable

(26) a. creer/soportar/comprender/vulnerar mucho/demasiado

b. *concebir/reparar/demostrar/describir mucho/demasiado

c. *sustituir/evitar/revocar/solucionar mucho/demasiado

Por último, cabe señalar que en unos pocos adjetivos en -ble parece haber una interpretación particular del elemento modal que impide la cuantificación del adjetivo, aun cuando las bases sean no delimitadas. Así, la imposibilidad de cuantificar indudable (*muy/demasiado indudable) se debe a una interpretación «estricta», delimitada, del elemento modal presente en la paráfrasis «literal»(de lo que no se puede dudar), pese a que el verbo dudar es [-delimitado] y, por lo tanto, cuantificable (p. ej., dudar mucho/demasiado ${ }^{14}$ ).

${ }_{13}$ Por su parte, las formas negativas creadas sobre adjetivos que no admiten la gradación, como los de (22b), mantienen las características de la base: cf. * muy desarmable, *muy inaplicable. Si en algún caso fuera posible la cuantificación, corresponderá siempre a la paráfrasis lexicalizada «(muy) difícil de V».

${ }^{14}$ Un ejemplo similar es comestible (*bastante/muy/demasiado comestible): poder (comer) tiene una interpretación delimitada, no gradual, pese a que es posible cuantificar el verbo base (comer mucho/ bastante/demasiado). 


\subsection{Adjetivos denominales}

Como se ha indicado en la sección 2.1., Morimoto 1998 señala que la diferenciación entre los rasgos [+delimitado] y [-delimitado] podría dar cuenta de los comportamientos disímiles de adjetivos relacionales y calificativos en cuanto a la posibilidad de recibir modificación de grado (véase ejemplos iniciales en 2).

Nótese que, en el caso de los adjetivos denominales, la oposición entre las dos clases de adjetivos aparece como una consecuencia directa de las características aspectuales del nombre base. Los adjetivos relacionales son mayoritariamente derivados, en general a partir de nombres de origen griego o latino, que se combinan con diversos sufijos, entre los que se puede destacar - al (y su variante alomórfica -ar) e -ico. Los ejemplos de (27) muestran que ninguno de esos adjetivos admite la cuantificación de grado con su significado relacional:

(27) a. (*muy) nominal/verbal/lunar/solar/fetal/bucal/teatral/estomacal

b. (*demasiado) lingüístico/semántico/telefónico/robótico/hepático

c. (*un poco) universitario/culinario/complementario/urbano/cutáneo/ ovino

A su vez, tampoco los nombres de los que provienen admiten cuantificadores singulares, ya que -en la interpretación relevante-se trata de nombres contables, como muestran los ejemplos de (28): si se les adjunta los cuantificadores de grado típicos de los nombres de masa (cf. ejemplos iniciales de 3-4) arroja un resultado agramatical.

(28) a. (*mucho/a) nombre/verbo/luna/sol/feto/boca/teatro/estómago

b. (*demasiado) lengua/significado/teléfono/robot/hígado

c. (*un poco de) universidad/cocina/complemento/ciudad/piel/ oveja

Este comportamiento es esperable si consideramos que los nombres contables tienen el rasgo [+delimitado] y que transmiten ese rasgo a los adjetivos derivados, que serán, por lo tanto, relacionales.

Por su parte, los adjetivos denominales que admiten la cuantificación de grado se forman mayoritariamente sobre nombres de masa. Como se puede advertir en los siguientes ejemplos, los adjetivos que admiten la modificación de grado en (29a) provienen todos de bases que reciben la cuantificación correspondiente a los nombres de masa (cf. 29b): 
(29) a (muy) envidioso/tramposo/carnoso/arenoso/culposo/aceitoso/ ruidoso $^{15}$

b. mucha/algo de envidia/trampa/arena/culpa/carne; mucho aceite/ruido

El sufijo -oso parece ser, con diferencia, el sufijo más productivo en este tipo de derivación, aunque puede mencionarse también el caso de -izo en pajizo o cobrizo).

Adjetivos calificativos de otro tipo, en cambio, se forman con los sufijos apreciativos -ón/a y -udo/a sobre nombres contables, como muestran los ejemplos de (30):

(30) a. (muy) culón/cabezón/narigón/panzón/tetona/bocona/huevón/ orejón

b. (muy) panzudo/orejudo/cabezudo/tetuda/dientudo/narigudo/ huesudo

Nótese que se trata de adjetivos graduables, no delimitados. El hecho de que puedan cuantificarse no es esperable de acuerdo con las correlaciones expuestas previamente, ya que están formados sobre nombres contables, con el rasgo [+delimitado]. Sin embargo, la cuantificación de grado modifica aquí a un elemento presente en la paráfrasis. Efectivamente, los adjetivos de (30) se parafrasean todos como «de $\mathrm{N}$ grande»; cuando aparece, el cuantificador de grado modifica, precisamente, a la cualidad referida al tamaño (que se expresa, no casualmente, mediante un adjetivo calificativo). Así, muy $\mathrm{N}$-ón o $\mathrm{N}$-udo (como en muy culón/panzudo) se leerá como «de N muy grande».

Otro caso similar es de los adjetivos que plantean una comparación implícita y que también pueden basarse en bases contables:

(31) (muy/bastante) aguileño/almendrado/gatuno/perruno

En los casos de (31) se pone en juego una comparación, que aparecerá explícita en la paráfrasis semántica: «similar a N», «del estilo de un $\mathrm{N}$ » $\mathrm{o}$ «como un $\mathrm{N}$ ». La cuantificación afecta, pues, a ese elemento comparativo.

Ahora bien, no en todos los sufijos que dan lugar a adjetivos denominales hay un predominio claro de la interpretación calificativa o relacional. También existen sufijos ambiguos, como en el caso de -ero/a: en general éste da lugar a adjetivos calificativos (32a), como sería espe-

\footnotetext{
15 Cabe destacar que -oso también da lugar a un número restringido de adjetivos relacionales basados en nombres de masa (nervioso, gaseoso, oleaginoso). Consideramos que en esos casos se aplica el análisis general propuesto para los adjetivos relacionales basados en nombres de masa (véanse, más adelante, los ejemplos 37-42).
} 
rable por el carácter predominantemente no contable de su base nominal, pero también puede formar adjetivos claramente relacionales (32b) o adjetivos que oscilan entre una y otra interpretación, como se desprende de la comparación de usos distintos del mismo adjetivo en (32c-d):

(32) a. un chico muy traicionero/casero/canchero/rockero/liero

b. la industria (*muy) pesquera/maderera/tabacalera/aceitera

c. un encuentro (*muy) callejero; un transporte (*muy) pasajero

d. un perro muy callejero; un problema medio pasajero

Luego presentaremos una explicación para los casos de (32b-c), cuyo comportamiento parece inesperado si tomamos como paradigmáticos de los adjetivos denominales en -ero los ejemplos de (32a).

Los casos que hemos visto hasta el momento se ajustan a la idea de que el rasgo [+/- delimitado] de la base determina el comportamiento de los adjetivos derivados. La única excepción encontrada (los adjetivos en -ón/a y-udo/a de 31) tiene una explicación convincente, basada en el adjetivo calificativo (y por lo tanto graduable) gran(de) que aparece en la paráfrasis. Sin embargo, hay otras situaciones «inesperadas» que deben tomarse en cuenta para una caracterización exhaustiva de los adjetivos denominales.

Por un lado, es sabido que un adjetivo relacional puede relexicalizarse en calificativo, como muestra el contraste entre (33) y (34); el proceso de relexicalización inversa, en cambio, es muy poco frecuente (cf., entre otros, Demonte 1999). En los casos de relexicalización, en los que aparece un significado diferente, el adjetivo sí aceptará la modificación de grado, como muestra (34). Nótese aquí que la presencia del cuantificador desambigua la interpretación semántica y la vuelve obligatoriamente calificativa:

(33) a. una obra (*muy/demasiado/terriblemente) teatral

b. un encuentro (*muy/demasiado/terriblemente) universitario

(34) a. un gesto muy/demasiado/terriblemente teatral

b. un gesto muy/demasiado/terriblemente universitario

En casos como (34), en los que se admite la cuantificación de grado, es evidente que la relación del adjetivo con el nombre base se ha desdibujado, ya que nuevamente aparece una interpretación comparativa: «típico/propio de un N», «al estilo de un N», «como (en) un N». En este contexto, no resulta extraño que un adjetivo derivado de un nombre contable pueda recibir modificación de grado: el cuantificador modifica en realidad al adjetivo calificativo (típico, propio) presente en la paráfrasis. 
Algo similar puede indicarse respecto de los gentilicios: en su significado «literal» no pueden recibir cuantificación de grado, puesto que se refieren a la pertenencia a un país o región (por definición, un nombre propio que tendrá el rasgo [+delimitado] $)^{16}$. En cambio, sí pueden hacerlo cuando la relación semántica con el nombre contable de base se difumina y aparecen significados como «típico/propio de N» (cf. Bosque 1990a):

(35) a. varios turistas (*muy) argentinos fueron detenidos en Barajas

b. un gesto muy argentino

El mismo tipo de observación se aplica a otros adjetivos formados originalmente sobre nombres propios. Así, los ejemplos de (36) tienen originariamente una interpretación agentiva: «de N», «compuesta/escrita por N» (cf. Bosque 1990a, Bosque y Picallo 1996); en esa interpretación no pueden recibir modificadores de grado, puesto que, evidentemente, tienen como base un nombre [+ delimitado] (cf. 36a). Sin embargo, de esa interpretación se deriva una interpretación calificativa «al estilo de $\mathrm{N}$ », como en (36b); en ese caso (y solo en ese caso) los adjetivos aceptan modificadores de grado, como muestra (36c) (cf. Bosque 1990a):

(36) a. obra wagneriana (= obra de Wagner) / drama cervantino (= drama de Cervantes)

b. obra wagneriana (= obra al estilo de W.)/drama cervantino (=drama al estilo de C.)

c. obra demasiado wagneriana/drama muy cervantino (sólo con la interpretación de b) ${ }^{17}$

En suma, el caso de adjetivos que se derivan de nombres contables, con el rasgo [+ delimitado], y que sin embargo admiten la modificación de grado, se explica porque la relexicalización del adjetivo relacional como calificativo entraña la aparición de un elemento cuantificable en la paráfrasis.

\footnotetext{
${ }^{16}$ Se excluyen de esta generalización los gentilicios intrínsecamente apreciativos como pajuerano.

17 Más habituales aún son los desplazamientos en el caso de marxista, peronista, cristiano, chomskyano y otros adjetivos con la interpretación de «partidario de (la doctrina/teoría de) X». La posibilidad o imposibilidad de cuantificar esos adjetivos depende directamente de la interpretación del elemento partidario presente en la paráfrasis: si se entiende que solo se puede ser o no partidario de una doctrina, estos adjetivos tendrán el rasgo [+delimitado] y no serán cuantificables. En cambio, si se entiende que puede haber grados en la pertenencia a una doctrina sí se admitirá la cuantificación del adjetivo: demasiado marxista, bastante cristiano, muy peronista.
} 
Como contrapartida, podemos ver que ciertos adjetivos relacionales están formados sobre bases que, de acuerdo con las pruebas vistas en (3-4), se clasifican como nombres de masa, como habíamos advertido para el caso de los ejemplos formados con -ero en (32b), que repetimos a continuación, junto con otros ejemplos:

(37) a. la industria (*muy) pesquera/maderera/tabacalera/aceitera

b. vida acuática/transporte aéreo/erupción cutánea

Este es un resultado inesperado en relación con las correlaciones establecidas previamente, ya que un adjetivo delimitado estaría formado sobre un nombre de masa, no delimitado. Sin embargo, la interpretación de esos adjetivos se basa en la determinación del nombre, como se advierte en las paráfrasis de (38). Al ser determinado, el nombre de masa pasa a tener el rasgo [+delimitado], lo cual explica la imposibilidad de que estos adjetivos reciban modificadores de grado, en forma paralela a los ejemplos de (27), formados sobre nombres contables (cf. 39):

(38) a. industria maderera = 'de la madera'

b. vida acuática $=$ 'en $e l$ agua'

c. problemas petroleros $=$ 'relativos a $l$ petróleo'

(39) a. *industria muy maderera

b. *vida demasiado acuática

c. *problemas un poco petroleros

El hecho de que los adjetivos relacionales siempre hacen alusión a una interpretación delimitada del nombre puede corroborarse en la imposibilidad de usar ese tipo de adjetivos en contextos que claramente remitan al nombre de masa. Esa imposibilidad se verifica, por ejemplo, si se trata de usar el adjetivo relacional en lugar de una construcción $d e+N$ en la que el nombre carezca de determinante, es decir, sea desnudo o escueto (cf. Bosque 1996), como se muestra en (40-41):

(40) a. vida en el agua $\square$ vida acuática

b. helado de agua $\boldsymbol{\square}$ *helado acuático

(41) a. industria de la madera $\mathbf{\square}$ industria maderera

b. mesa de madera $\boldsymbol{\square} *$ mesa maderera

La obligatoriedad de parafrasear los adjetivos relacionales derivados de nombres de masa por medio de sintagmas de determinante es aún más obvia en el caso de los adjetivos relacionales temáticos, de acuerdo con la distinción de Bosque y Picallo 1996. Los ejemplos de (42a), que saturan, en cada caso, el requerimiento de tema, locativo o agente del 
verbo base de la nominalización, requieren obligatoriamente la presencia del determinante al ser parafraseados por una construcción preposicional, como se advierte en (42b):

(42) a. contaminación acuática/erupción cutánea/pesca japonesa

b. 'contaminación de $l$ agua'/'erupción en la piel'/ pesca de los japoneses'

Cabe señalar, por último que, en ocasiones, una misma base nominal da lugar a dos adjetivos, uno relacional y el otro calificativo, como puede advertirse en los pares de (43):

(43) a. (*muy) acuático vs. muy acuoso

b. (*muy) aceitera vs. muy aceitosa

c. (*muy) dental vs. muy dientudo

d. (*muy) óseo vs. muy huesudo

En estos casos, con independencia de la naturaleza del nombre, sistemáticamente el adjetivo relacional tendrá una paráfrasis que supone la aparición del determinante (p. ej., en/de/relativo al agua/el hueso/los dientes/el aceite) y, por lo tanto, la asignación del rasgo [+delimitado] al nombre de masa. Por su parte, el correspondiente adjetivo calificativo supone la lectura [-delimitado]. La posibilidad de cuantificación de grado se explica, así, o bien por el carácter no delimitado del nombre base (que se mantiene en la paráfrasis: de/con/como agua/aceite), como en los ejemplos relevantes de (42a-b), o bien por la presencia de algún adjetivo calificativo en una paráfrasis más compleja (de dientes/huesos grandes), pese a que el nombre base es contable, como en (42c-d).

\section{Conclusiones}

Desde el punto de vista empírico, a lo largo de este trabajo hemos mostrado que una categorización básica de las palabras simples y derivadas se establece a partir de la distinción entre los rasgos [+delimitado] y [-delimitado]. En el caso particular de los adjetivos analizados aquí, la distinción resulta más primaria que etiquetas como «calificativo» o «relacional», en la medida en que afecta también a adjetivos que suelen permanecer al margen de esa oposición, como los adjetivos deverbales. Por lo demás, la cuantificación se ha revelado como una propiedad sintáctica consistente a los fines de establecer la diferenciación entre adjetivos delimitados y no delimitados.

De la descripción y el análisis de los adjetivos derivados que hemos emprendido en el presente trabajo puede extraerse una generalización relevante, que es la siguiente: 
(I) La base determina el rasgo [+/-delimitado] de los adjetivos derivados, que se verifica en la posibilidad o imposibilidad de cuantificación de $\operatorname{grado}^{18}$.

Hemos visto en este trabajo que el rasgo [+/-delimitado] de la base parece pasible de ser modificado en diversos casos de lexicalización de las formas. Se puede advertir en este punto una asimetría entre los adjetivos deverbales y los denominales. En efecto, la única excepción sistemática entre los adjetivos deverbales se da con algunos adjetivos en -ble, que pueden admitir la cuantificación aunque provengan de un verbo delimitado o télico, dado que el cuantificador afecta al elemento modal implícito en el significado del sufijo (p. ej. fácil).

En el caso de los adjetivos denominales, en cambio, la situación es más compleja, probablemente a causa del doble sistema de cuantificación señalado en los ejemplos iniciales de (3-4). Un número importante de adjetivos denominales, tanto relacionales como calificativos, parecen atenerse a la generalización de (I). Otros, en cambio, se muestran como excepciones. Así, por ejemplo, pueden derivarse adjetivos graduables a partir de nombres contables si los sufijos agregan a la base un significado no delimitado. Ese significado puede referirse al tamaño (como en los sufijos -udo/-ón), o bien aludir a alguna comparación o analogía (como en los sufijos -ado/-uno). En ambos casos, la cuantificación afectará a los diversos elementos semánticos implícitos en la paráfrasis correspondiente (grande, similar a, típico de o propio de). Algo semejante sucede en los adjetivos relacionales que se relexicalizan como calificativos, perdiendo la relación directa con el nombre contable que les sirve de base, en cuyo caso pueden ser graduables, como teatral o universitario. Por su parte, el proceso inverso se verifica en el caso de aquellos adjetivos relacionales en los que el nombre de base es de masa (i.e., no delimitado), como en los casos de petrolera o acuática. Concluimos que en esos casos el nombre se ha recategorizado a partir de la determinación que se explicita en la paráfrasis (de $l$ petróleo, en $e l$ agua) y por eso puede dar lugar a un adjetivo delimitado, no graduable.

Cabe recordar que también en la herencia de propiedades argumentales se advierten modificaciones entre la estructura argumental de la base y la del derivado: por ejemplo, los adjetivos en -ble no heredan, por su significado (medio pasivo), el argumento externo que es propio de los verbos transitivos de los que derivan (cf. Gràcia 1995). La existencia de casos en los que no se produce la herencia directa del rasgo [+/-delimitado] de la base al derivado, entonces, no resta valor a la generalización (I), que se verifica en la amplia mayoría de los casos.

18 Plausiblemente, la generalización de (A) podría extenderse a todos los casos de derivación con cambio categorial, aunque esa pretensión excede los límites del presente trabajo. 


\section{REFERENCIAS BIBLIOGRÁFICAS}

ABNEY, S. (1987): The English noun phrase in its sentential aspect, tesis doctoral inédita, Cambridge, Mass., MIT.

BosQue, I. (1990a): Las categorías gramaticales. Relaciones y diferencias, Madrid, Síntesis.

—, (1990b): Tiempo y aspecto en español, Madrid, Cátedra.

—, (1996): «Por qué determinados sustantivos no son sustantivos determinados. Repaso y balance», en Bosque, I. (ed.), El sustantivo sin determinación, Madrid, Visor, pp. 13-119.

—, (1999a): «El nombre común» en Bosque, I. y Demonte, V. (eds.), Gramática descriptiva de la lengua española, Vol. I, Madrid, Espasa-Calpe, pp. 3-77.

—, (1999b): «El sintagma adjetival. Modificadores y complementos del adjetivo», en Bosque, I. y Demonte, V. (eds.), Gramática descriptiva de la lengua española, Vol. I, Madrid, Espasa-Calpe, pp. 217-310.

—, (2001): «Sobre la gramática de la gradación», en Valencia, A. (ed.), Sociedad Chilena de Lingüistica. Un aniversario muy especial, Santiago de Chile, LOM Ediciones, pp. 39-79.

- y Masullo, P. (1997): «On verbal quantification in Spanish», Proceedings of the Third workshop on the syntax of central Romance languages, Girona, pp. 9-63.

- y Picallo, C. (1996): «Postnominal adjectives in spanish DPs», Journal of Linguistics 32, pp. 349-385.

Bresnan, J. (1978): «A realistic transformational grammar», en Halle, M., Bresnan, J. y Miller, G. (eds.), Linguistic theory and psychological reality, Cambridge, Mass., MIT Press, pp. 1-59.

Demonte, V. (1999): «El adjetivo: clases y usos. La posición del adjetivo en el sintagma nominal», en Bosque, I. y Demonte, V. (eds.), Gramática descriptiva de la lengua española, Vol. I, Madrid, Espasa-Calpe, pp.129-216.

De Miguel, E. (1986): «Sulla regola di formazione degli aggettivi in -ble in spagnolo», Rivista di Grammatica Generativa 11, pp. 127-165.

DowTy, D. (1979): Word meaning and montague grammar, Dordrecht, Reidel.

GRÀCIA, Ll. (1995): Morfologia lèxica. L'herència de l'estructura argumental, Valencia, Universitat de València.

—; Cabré, M. T.; VAREla, S.; Azkarate, M. et al. (eds.) (1999): Configuración morfológica y estructura argumental: léxico y diccionario, Guipuzkoa, Universidad del País Vasco.

Hale, K. y Keyser, S. (1993): «On the argument structure and the lexical expression of syntactic relations», en Hale, K. y Keyser, S. (eds.), A view from Building 20 th , Cambridge, Mass., MIT Press, pp. 53-109.

-, (2002): Prolegomenon to a theory of argument structure, Cambridge, Mass., MIT Press.

JACKENDOFF, R. (1991): «Parts and boundaries», Cognition 41, pp. 9-45.

KoRnFeld, L. (2007): «Sobre la distribución del afijo -it $(o / a)$ con adjetivos en español», IV Encuentro de Gramática Generativa, Instituto de Ciencias Humanas Sociales y Ambientales (Incihusa), Mendoza, 26 al 28 de julio de 2007. 
- y RESNIK, G. (2002): «Estructura argumental y propiedades categoriales: el caso de los adjetivos participiales», Actas del IX congreso de la Sociedad Argentina de Lingüística (en CD), Córdoba, Universidad Nacional de Córdoba, 2003.

LACA, B. (1993): «Las nominalizaciones orientadas y los derivados españoles en -dor y -nte», en Varela, S. (ed.), La formación de palabras, Madrid, Taurus, pp. 180-204.

LEVIN, B. y RAPPAPORT, M. (1986): «The Formation of adjectival passives», Linguistic Inquiry 17, pp. 623-661.

LIEBER, R. (1983): «Argument linking and compounding in English», Linguistic Inquiry 14, pp. 251-286.

—, (1992): Deconstructing morphology, Chicago, University of Chicago Press.

Morimoto, Y. (1998): El aspecto léxico, Madrid, Arco/Libros.

RAINER, F. (1999): «La derivación adjetival», en Bosque, I. y Demonte, V. (eds.), Gramática Descriptiva de la Lengua Española, Vol. III, Madrid, Espasa, pp. 45954643.

ROEPER, T. (1987): «Implicit arguments and the head-complement Relation», Linguistic Inquiry 18, pp. 267-310.

VENDLER, Z. (1957): «Verbs and times», The Philosophical Review 66, 2, pp. 143-160. 